\title{
Construção da Carreira: O Papel da Perceção dos Filhos acerca dos Estilos Educativos Parentais na Exploração Vocacional
}

\author{
Career Construction: The Role of Children's Perception about Parental \\ Educational Styles in Vocational Exploration
}

\author{
Liliana Costa Faria ${ }^{*}, a$, Joana Carneiro Pinto $^{b} \&$ Matilde Vieira $^{c}$ \\ ${ }^{a}$ Universidade Europeia, Lisboa, Portugal, ${ }^{b}$ Universidade Católica Portuguesa, Lisboa, Portugal \\ ${ }^{c}$ ISLA Leiria, Leiria, Portugal
}

\begin{abstract}
Resumo
Este trabalho analisa os estilos educativos parentais percebidos pelos jovens e os seus níveis de exploração vocacional, atendendo aos referenciais conceptuais da teoria da construção da carreira e à investigação prévia sobre a relação entre a influência parental e o desenvolvimento vocacional. Participam 296 estudantes portugueses, 109 (36,82\%) do sexo feminino e 187 (63,18\%) do sexo masculino, com uma idade média de 17,03 anos $(D P=1,45 ; \operatorname{Min}=14 ; \operatorname{Max}=23)$, que preencheram a Escala de Exploração Vocacional e o Questionário de Estilos Educativos Parentais. Verificaram-se diferenças na percepção do estilo educativo parental e na exploração vocacional em função de variáveis sociodemográficas, e uma associação positiva significativa entre a exigência e as crenças e os comportamentos de exploração vocacional. Discutem-se implicações acerca da participação parental na construção de carreira.

Palavras-chave: Construção da carreira, exploração vocacional, estilos educativos parentais, jovens.
\end{abstract}

\begin{abstract}
This work analyzes the parental educational styles perceived by youth and their level of vocational exploration, considering the career construction theory and previous research on the relationship between parental influence processes and vocational development. Participants were 296 adolescents, $109(36.82 \%)$ girls and 187 (63.18\%) boys, with a mean age of 17.03 years $(S D=1.45$; Min. $=14$; Max. $=23$ ) who fulfilled the Career Exploration Survey and the Parental Educational Styles Questionnaire. Results show differences in the perceived parental educational styles and career exploration according to socio demographic variables, and a significant positive association between requirement and career exploration beliefs and behaviors. Implications about the parental involvement in career construction are discussed.

Keywords: Career construction, career exploration, parental educational styles, youth.
\end{abstract}

O presente estudo visa aprofundar o conhecimento das perceções dos jovens acerca dos estilos parentais dos seus pais, nas suas construções de carreira, através da avaliação do estilo educativo parental percecionado pelos filhos, bem como, dos seus níveis de exploração vocacional. Assim, analisa-se a teoria da construção da carreira de Savickas $(2002,2005)$, que se centra na interpretação e no processo interpessoal a partir do qual os indivíduos dão significado e sentido ao seu comportamento vocacional. E, atende-se à investigação sobre a relação entre os processos de influência parental e o desenvolvimento vocacional (e.g., Schulenberg, Vondracek, \& Crouter, 1984; Whiston \& Keller, 2004). Pretende-se com este trabalho contribuir para a compreensão da relação entre influência dos estilos educativos parentais e o comportamento exploratório voca-

\footnotetext{
"Endereço para correspondência: Quinta do Bom Nome, Lisboa, Portugal 1500-210. E-mail: lilianafaria@delphis. com.pt
}

cional, campo da orientação profissional cujo investimento tem sido escasso e limitado.

\section{A Teoria da Construção da Carreira de Savickas}

A teoria da construção da carreira (Savickas, 2002, 2005), projetada para ser utilizada em sociedades multiculturais e economias globais, fornece uma explicação contemporânea da carreira e explica os processos interpretativos e interpessoais através dos quais as pessoas se constroem, impondo direção ao seu comportamento vocacional, e dando sentido às suas carreiras. Esta teoria assenta no construtivismo pessoal e no construcionismo social, defendendo que as pessoas constroem representações da realidade, mas não constroem a própria realidade. Além disso, esta teoria concebe a carreira a partir de uma perspetiva contextualista, na qual concetualiza o desenvolvimento como impulsionado pela adaptação a um ambiente, em vez de uma sequência de papéis e tarefas que cada pessoa vai assumindo ao longo do seu ciclo de 
Faria, L. C., Pinto, J. C. \& Vieira, M. (2015). Construção da Carreira: O Papel da Perceção dos Filhos acerca dos Estilos Educativos Parentais na Exploração Vocacional.

vida, em função do grau de maturação das suas estruturas internas (Savickas, 2005).

Assim sendo, as carreiras são construídas à medida que as pessoas fazem as suas escolhas, e nelas expressam os seus autoconceitos e objetivos pessoais (Savickas, 2005). Isto é, as pessoas constroem a sua carreira impondo significados ao seu comportamento vocacional e às suas experiências ocupacionais. A carreira é, por isso, fruto de uma construção subjetiva que implica atribuir um significado pessoal a memórias passadas, experiências presentes, e aspirações futuras, tecendo-as num tema de vida que compõe o padrão da vida de trabalho da pessoa (Savickas, 2005).

Ainda segundo esta teoria, as pessoas, através das suas ações na família, compõem um papel social como atores, e depois adaptam esse papel para usar nos múltiplos palcos em que se movem, nomeadamente, na escola e na comunidade e, eventualmente, como autores de histórias autobiográficas que explicam a continuidade e coerência em experiências ocupacionais (Savickas, 2005). Deste modo, os processos de autoconstrução da carreira diferem durante a infância, adolescência, e idade adulta. Os indivíduos começam a autoconstrução como atores e, mais tarde, tornam-se agentes de ação direta e, por fim, tornam-se autores que explicam essas mesmas ações (McAdams \& Olson, 2010).

\section{A Influência Parental na Construção da Carreira}

$\mathrm{O}$ tema da influência parental no desenvolvimento vocacional de jovens tem sido um dos aspetos mais recentemente valorizados na literatura vocacional e educacional mais geral, sendo unânime a importância da família no desenvolvimento de carreira dos jovens (e.g., Schulenberg et al., 1984; Whiston \& Keller, 2004). Vários estudos demonstram que o desenvolvimento de carreira dos adolescentes é influenciado pela qualidade da relação filhos-pais (Blustein, Walbridge, Friedlander, \& Palladino, 1991; O’Brien, 1996; Scott \& Church, 2001; Vignoli, Croity-Belz, Chapeland, Fillipis, \& Garcia, 2005; Whiston \& Keller, 2004), constituindo quer, os estilos parentais dos pais, quer as perceções por parte dos filhos sobre esses mesmos estilos parentais, bons preditores do desenvolvimento da carreira (Penick \& Jepsen, 1992).

A interação pais-filhos tem sido descrita na literatura como uma variável processual de importância no desenvolvimento da carreira (Schulenberg et al., 1984) uma vez que os pais são a principal fonte de conhecimento, crenças e valores para as crianças e jovens (Bryant, Zvonkovic, \& Reynolds, 2006; Otto, Markman, Gureckis, \& Love, 2010; Otto, 2000), e que a interação pais-filhos envolve a comunicação, o suporte, o encorajamento e a orientação (Berríos-Allison, 2005; Carvalho, 2007; Gonçalves, 2006; Pinto \& Soares, 2002; Schultheiss \& Blustein, 1994; Young et al., 2005). Por exemplo, Kohn (1977) e Mortimer (1976) sublinham que os filhos tendem a fazer opções escolares e profissionais em conformidade com os valores da família de origem.
Além disso, com a finalidade de obter conhecimento sobre o trabalho dos adultos, as crianças e adolescentes tendem a basear-se na observação direta dos seus pais no trabalho ou através de conversas que ouvem sobre o trabalho dos pais. As experiências e as relações informais, incluindo as ocorridas em contexto familiar, constituem-se como recursos ou obstáculos para o processo de implementação da tomada de decisão (Galinsky, 2000). Estudos prévios (Otto et al., 2010; Otto, 2000) demonstram, igualmente, que os adolescentes falam mais frequentemente acerca de assuntos de carreira com os seus pais e nomeiam estes como sendo os que exerceram uma maior influência durante as transições de carreira, através da relação que com eles estabelecem.

O papel dos pais na construção da carreira dos filhos processa-se ao nível da construção de uma identidade, da exploração vocacional, da formulação de preferências vocacionais, e da tomada de decisão (Dietrich \& Kracke, 2009; Whiston \& Keller, 2004) e, da implementação de uma decisão por parte dos adolescentes (Fouad et al., 2008), designadamente através do suporte (Carvalho, 2007; O’Brien, 1996) e da afetuosidade (Noack, Kracke, Gniewosz, \& Dietrich, 2010).

\section{Estilos Educativos Parentais e Influência Parental na Construção da Carreira}

De entre as diferentes variáveis associadas às interações pais-filhos, os estilos educativos parentais, ou seja, o padrão de comportamento dos pais, criado pelo conjunto das suas atitudes, no qual se inclui as práticas disciplinares/ educativas parentais (Darling \& Steinberg, 1993), parecem constituir-se como uma dimensão do comportamento parental com capacidade de influenciar o comportamento dos jovens (Ducharne, Cruz, Marinho, \& Grande, 2006; Hutz \& Bardagi, 2006).

Segundo, Baumrind (1971) e, Maccoby e Martin (1983), os estilos educativos parentais, subdividem-se em duas dimensões ortogonais: capacidade de resposta às solicitações dos filhos (responsividade) versus, atitudes de exigência (exigências).

A responsividade parental refere-se às atitudes de compreensão, de apoio emocional e de comunicação bidirecional dos pais para com os filhos (Costa, Teixeira, \& Gomes, 2000; Teixeira, Bargadi, \& Gomes, 2004). Em contraste, a exigência parental, refere-se ao controlo/regulação do comportamento e ao estabelecimento de metas e padrões de conduta por parte dos pais, o que inclui todas as atitudes dos pais que procuram de alguma forma controlar o comportamento dos filhos, impondo aos filhos, limites e estabelecendo regras.

A investigação no domínio da influência parental no desenvolvimento vocacional sugere, por exemplo, que o estilo parental democrático (Baumrind, 1971, 1989), combinando exigência e responsividade, proporciona um clima familiar harmonioso, promove a independência do jovem, e favorece o estabelecimento de objetivos, o que cria condições para uma exploração vocacional mais ativa 
(Kracke, 1997). Ainda neste sentido, o estudo levado a cabo por Way e Rossmann (1996) revelou que este estilo democrático influenciaria a construção de um ambiente familiar proativo, que ajudaria os jovens a tornarem-se mais autónomos e responsáveis. Este estilo criaria ainda condições para a transmissão de valores laborais, e para aprendizagens no domínio da tomada de decisão, hábitos de trabalho, resolução de conflitos e competências de comunicação. Holland (1992) verificou a existência de relações significativas entre participantes do sexo masculino com aspirações ligadas ao domínio Investigador e figuras parentais que revelavam um estilo parental democrático. Neste sentido, pais democráticos, que encorajam os filhos à exploração e discussão de informação pertinente às suas tomadas de decisão, promovem nos filhos interesses por atividades intelectuais, científicas e académicas, nas quais são estimulados a utilizar as suas competências intelectuais para compreender fenómenos físicos, biológicos e culturais. Por seu lado, um estilo parental autoritário (Baumrind, 1971, 1989), ou seja, altamente exigente mas não responsivo, pressiona os jovens a conformarem-se e realizar as expectativas dos pais relativamente à educação e à carreira, o que poderá levar a uma fraca correspondência entre o indivíduo e a escolha vocacional, bem como, a condições diminuídas de saúde mental e relações familiares perturbadas. Holland (1992) verificou ainda a existência de associações significativas entre as aspirações Realistas, dos participantes do sexo masculino, e pais e mães com estilos educativos parentais marcados pelo autoritarismo. Ou seja, pais que têm um conjunto de atitudes restritivas e controladoras face aos seus filhos, e que exigem o cumprimento rígido de um conjunto de normas, geralmente promovem nos seus filhos interesses por atividades mais objetivas, práticas, físicas e de manipulação sistemática de objetos, ferramentas, máquinas, animais ou plantas. Finalmente, os pais que adotam um estilo parental permissivo (Baumrind, 1971, 1989), isto é, com baixa exigência e baixa responsividade, tendem a mostrar-se pouco acessíveis no atendimento dos interesses da criança, em termos de pessoas e objetos, o que dificulta o desenvolvimento do conhecimento de si própria, bem como a diferenciação dos seus objetivos de carreira, daqueles que são expressos pelos seus pais.

O estudo conduzido por Vignoli et al. (2005) revelou que para as participantes do sexo feminino, do ensino secundário, níveis elevados de ansiedade generalizada, e autorrelatos de um estilo parental negligente, estavam negativamente relacionados com a exploração vocacional. Relativamente, aos participantes do sexo masculino, os resultados mostraram que o medo de desiludir os pais estava positivamente relacionado com a exploração vocacional (Vignoli et al., 2005).

Tendo em conta o supra referido, os objetivos prosseguidos neste estudo foram analisar a diferenciação, em função do sexo, dos níveis de exploração vocacional e dos estilos educativos parentais percecionados e, analisar a relação entre os estilos educativos parentais perceciona- dos e os níveis de exploração vocacional, possibilitando o desenho de possíveis estratégias intencionais dirigidas para a rentabilização/otimização dos recursos relacionais da família na construção da carreira dos adolescentes.

\section{Metodologia}

\section{Amostra}

Participaram neste estudo 296 adolescentes, de ambos os sexos $(109,36,80 \%$ do sexo feminino e $187,63,20 \%$ do sexo masculino), com idades compreendidas entre os $14 \mathrm{e}$ os 23 anos $(M=17,03 ; D P=1,45)$, provenientes de duas escolas públicas da região centro de Portugal. Dos 296 adolescentes, 83 (28\%) frequentam o ensino secundário regular $\left(10^{\circ}\right.$ ano $=62,21 \% ; 12^{\circ}$ ano $\left.=21 ; 7,10 \%\right)$ e 213 (72\%) frequentam o ensino secundário profissional $\left(1^{\circ}\right.$ ano $=112 ; 37,80 \% ; 2^{\circ}$ ano $\left.=101 ; 34,10 \%\right)$.

A maioria dos encarregados de educação possui, como habilitações literárias, entre o nível do ensino básico $\left(1^{\circ}\right.$ ciclo) e do ensino secundário completo, destacando-se as habilitações literárias dos encarregados de educação ao nível do ensino básico ( $9^{\circ}$ ano de escolaridade), com cerca de $36,50 \%(n=108)$, seguidos de $22,60 \%(n=67)$ com o ensino secundário completo.

A situação profissional dos encarregados de educação é na maioria com emprego $82 \%(n=243)$ e apenas $18 \%$ $(n=53)$ de desempregados.

\section{Instrumentos}

Para a avaliação dos estilos educativos parentais e a exploração vocacional foram aplicados o Questionário de Estilos Educativos Parentais (Lamborn, Mounts, Steinberg, \& Dornbusch, 1991; QEEP, adapt. por Ducharne et al., 2006) e a Escala de Exploração Vocacional (Stumpf, Colarelli, \& Hartman, 1983; EEV, adapt. por Taveira, 1997), respetivamente.

O Questionário de Estilos Educativos Parentais (Lamborn et al., 1991; QEEP, adapt. por Ducharne et al., 2006) é composto por 19 itens que avaliam as perceções que os jovens adolescentes têm dos estilos educativos dos seus pais, partindo das dimensões de responsividade/afeto/ envolvimento ( 10 itens) e a supervisão/exigência ( 9 itens). A responsividade abrange as atitudes e comportamentos parentais de compreensão, apoio emocional, e comunicação bidirecional, destinados a promover a autonomia e autoafirmação dos seus filhos na satisfação das suas necessidades. A exigência envolve as atitudes e comportamentos parentais de controlo, supervisão e disciplina, destinados a promover o seguimento das normas, regras e padrões de conduta.

Nos cinco primeiros itens, o pai e a mãe são avaliados separadamente, enquanto nos restantes itens são avaliados em simultâneo. O questionário apresenta bons índices de consistência interna com alfa de Cronbach, de 78 para a responsividade e 85 para a exigência (Ducharne et al., 2006).

A Escala de Exploração Vocacional (Stumpf et al., 1983; EEV, adapt. por Taveira, 1997) é constituída por 53 
Faria, L. C., Pinto, J. C. \& Vieira, M. (2015). Construção da Carreira: O Papel da Perceção dos Filhos acerca dos Estilos Educativos Parentais na Exploração Vocacional.

itens que avaliam as crenças (Estatuto de Emprego, Certeza nos Resultados da Exploração, Instrumentalidade Externa, Instrumentalidade Interna, Importância de obter a Posição Preferida), os comportamentos (Exploração Orientada para o Meio, Exploração orientada para Si Próprio/a, Exploração Intencional-Sistemática, Quantidade de Informação), e as reações afetivas (Satisfação com a Informação, Stresse na Exploração, Stresse na Tomada de Decisão) relacionadas com o processo de exploração de carreira

O Estatuto de Emprego avalia até que ponto são favoráveis, as possibilidades de emprego na área preferida; a Certeza nos Resultados da Exploração mede o grau de convicção para atingir uma posição favorável no mercado de trabalho; a Instrumentalidade Externa estima o valor instrumental atribuído à exploração do mundo profissional; a Instrumentalidade Interna avalia o valor instrumental da exploração orientada para si próprio/a; a Importância de Obter a Posição Preferida avalia o grau de importância atribuído à realização da preferência vocacional; a Exploração Orientada para o Meio mede o grau de exploração de profissões, empregos, e organizações, realizada nos últimos três meses; a Exploração Orientada para Si Próprio/a avalia o grau de exploração pessoal e de retrospeção realizada nos últimos três meses; a Exploração Intencional-Sistemática analisa em que medida a procura de informação sobre o meio e sobre si próprio/a se realizou de um modo intencional e sistemático; a Quantidade de Informação Obtida mede a quantidade de informação adquirida sobre as profissões, empregos e organizações e sobre si próprio/a; a Satisfação com a Informação Obtida estima em que medida a informação obtida sobre as profissões, os empregos e as organizações de trabalho mais relacionadas com os seus interesses, capacidades e necessidades proporciona satisfação; o Stresse na Exploração analisa o grau de stresse antecipado face a nova exploração, por comparação a outros acontecimentos de vida; o Stresse na Tomada de Decisão analisa o stresse antecipado face à tomada de decisão, por comparação a outros acontecimentos.

$\mathrm{O}$ estudo de análise fatorial confirmatória do CES realizado por Taveira (1997) permite evidenciar a robustez deste modelo de doze dimensões consistentes de exploração vocacional, para ambos os sexos e dois anos de escolaridade $\left(9^{\circ}\right.$ e $12^{\circ}$ anos, $\left.N=1400\right)$.

\section{Procedimentos}

Para a realização deste estudo, foi inicialmente obtida a autorização junto dos autores dos instrumentos para a sua utilização. De seguida, foi estabelecido um contacto formal com as escolas que colaboraram no estudo. O processo de recolha de dados decorreu entre os meses de maio e junho de 2011. Foram solicitadas as autorizações junto dos encarregados de educação, aos alunos com menos de 18 anos e reforçado o caráter voluntário de participação. Foram igualmente garantidas todas as recomendações éticas de confidencialidade dos dados, reforçando-se que seriam utilizados unicamente para fins desta investigação. Não foi feita qualquer submissão a Comitê de Ética, dado não ser um padrão requerido em Portugal. Os dados depois de recolhidos foram analisados com recurso a análises de estatística descritiva, bem como a análises de estatística inferencial, nomeadamente, testes $t$ de Student e correlações de Pearson. Os resultados foram considerados estatisticamente significativos quando o valor do teste de significância foi inferior a $0,05(p<0,05)$.

\section{Resultados}

\section{Exploração e Perceção dos Estilos Educativos Parentais em Função do Sexo}

A Tabela 1 apresenta a média e desvio-padrão para as subescalas de exploração vocacional e de estilos educativos parentais, para os participantes do sexo feminino e masculino, bem como, as diferenças estatisticamente significativas para estas variáveis, em função do sexo.

Os valores na perceção dos estilos educativos parentais oscilam entre os obtidos na dimensão Exigência $(M=$ $26,49 ; D P=3,19)$ e os alcançados na dimensão Responsividade $(M=27,27 ; D P=5,74)$. Verificou-se, igualmente, que os resultados estão acima do ponto médio em todas as subescalas, quer na amostra total, quer nas amostras dos participantes do sexo feminino e masculino.

Os níveis de exploração vocacional oscilam entre os valores obtidos na subescala Exploração Sistemática Intencional $(M=5,27 ; D P=1,86)$ e os alcançados na subescala Instrumentalidade Externa $(M=36,11 ; D P=6,34)$. Verificou-se, igualmente, que os resultados alcançados são iguais ou superiores ao ponto médio em praticamente todas as subescalas, considerando o sexo. As exceções verificam-se nas subescalas Certeza de Resultados de Exploração, Importância da Posição Preferida, Exploração do Meio, Exploração Sistemática Intencional e Stresse com a Exploração, em que ambos os sexos obtiveram resultados inferiores ao ponto médio da respetiva subescala. $\mathrm{Na}$ subescala do Stresse com a Decisão para os rapazes também se obteve um resultado inferior ao ponto médio.

Tal como se pode verificar pela leitura da Tabela 1, constata-se a existência de uma diferença estatisticamente significativa ao nível da Exigência $(t=2,20 ; p<0,05)$, favorável às participantes do sexo feminino. Além disso, verifica-se, ainda, a existência de uma diferença estatisticamente significativa ao nível das subescalas Instrumentalidade Externa $(t=3,23 ; p<0,05)$, Instrumentalidade Interna $(t=3,21 ; p<0,05)$, Exploração de Si Próprio $(t=2,82$; $p<0,05)$, favorável às participantes do sexo feminino, e Exploração Sistemática Intencional $(t=-2,36 ; p<0,05)$, favorável aos participantes do sexo masculino.

\section{Perceção dos Estilos Educativos Parentais dos Jovens e Exploração Vocacional}

Tendo em vista o aprofundamento do conhecimento das associações entre o processo de exploração vocacional e as perceções dos estilos educativos parentais dos jovens, procedeu-se, à análise das correlações entre as subescalas das crenças, comportamento e reações de exploração vocacional e entre as dimensões Exigência e Responsividade. 
Tabela 1

Exploração e Perceção dos Estilos Educativos Parentais em Função do Sexo

\begin{tabular}{|c|c|c|c|c|c|}
\hline & Sexo & $N$ & $M$ & $D P$ & $T$ \\
\hline \multirow{2}{*}{ EE } & Feminino & 109 & 9,39 & 2,44 & \multirow{2}{*}{$-0,15$} \\
\hline & Masculino & 187 & 9,43 & 2,22 & \\
\hline \multirow{2}{*}{ CRE } & Feminino & 109 & 8,15 & 3,15 & \multirow{2}{*}{$-0,80$} \\
\hline & Masculino & 187 & 8,42 & 2,66 & \\
\hline \multirow{2}{*}{ IE } & Feminino & 109 & 37,65 & 6,56 & \multirow{2}{*}{$3,23 *$} \\
\hline & Masculino & 187 & 35,22 & 6,50 & \\
\hline \multirow{2}{*}{ II } & Feminino & 109 & 14,76 & 2,85 & \multirow{2}{*}{$3,21 *$} \\
\hline & Masculino & 187 & 13,64 & 2,92 & \\
\hline \multirow{2}{*}{ IPP } & Feminino & 109 & 11,55 & 2,43 & \multirow{2}{*}{1,91} \\
\hline & Masculino & 187 & 10,99 & 2,43 & \\
\hline \multirow{2}{*}{ EM } & Feminino & 109 & 11,45 & 3,42 & \multirow{2}{*}{1,21} \\
\hline & Masculino & 187 & 10,95 & 3,41 & \\
\hline \multirow{2}{*}{ ESP } & Feminino & 109 & 15,53 & 3,87 & \multirow{2}{*}{$2,82 *$} \\
\hline & Masculino & 187 & 14,18 & 4,06 & \\
\hline \multirow{2}{*}{ ESI } & Feminino & 109 & 4,94 & 1,82 & \multirow{2}{*}{$-2,36^{*}$} \\
\hline & Masculino & 187 & 5,46 & 1,86 & \\
\hline \multirow{2}{*}{ QI } & Feminino & 109 & 9,91 & 1,90 & \multirow{2}{*}{1,37} \\
\hline & Masculino & 187 & 9,59 & 1,97 & \\
\hline \multirow{2}{*}{ SI } & Feminino & 109 & 9,96 & 1,87 & \multirow{2}{*}{1,69} \\
\hline & Masculino & 187 & 9,57 & 1,95 & \\
\hline \multirow{2}{*}{ SE } & Feminino & 109 & 15,42 & 5,45 & \multirow{2}{*}{1,74} \\
\hline & Masculino & 187 & 14,40 & 4,57 & \\
\hline \multirow{2}{*}{ SD } & Feminino & 109 & 21,05 & 7,30 & \multirow{2}{*}{1,50} \\
\hline & Masculino & 187 & 19,81 & 6,53 & \\
\hline \multirow{2}{*}{ Exigência } & Feminino & 109 & 27,02 & 3,09 & \multirow{2}{*}{$2,21^{*}$} \\
\hline & Masculino & 187 & 26,18 & 3,21 & \\
\hline \multirow{2}{*}{ Responsividade } & Feminino & 109 & 27,99 & 5,81 & \multirow{2}{*}{1,66} \\
\hline & Masculino & 187 & 26,85 & 5,67 & \\
\hline
\end{tabular}

${ }^{*} p<0,05$.

A leitura da Tabela 2 permite identificar a presença de uma correlação positiva forte entre a subescala Estatuto de Emprego e as subescalas de Certeza nos Resultados de Exploração $(r=0,50 ; p<0,01)$; Instrumentalidade Externa $(r=0,42 ; p<0,01)$; Instrumentalidade Interna $(r$ $=0,32 ; p<0,01)$; Importância da Profissão Preferida $(r=$ $0,23 ; p<0,01)$. Verifica-se a presença de uma correlação positiva forte entre a subescala Certeza dos Resultados de Exploração e as subescalas de Instrumentalidade Externa $(r=0,33 ; p<0,01)$; Instrumentalidade Interna $(r=0,19$; $p<0,01)$; Importância da Posição Preferida $(r=0,22 ; p<$ $0,01)$. Verifica-se também, a presença de uma correlação positiva forte entre a subescala Instrumentalidade Externa e as subescalas Instrumentalidade Interna $(r=0,66 ; p<$ 
Faria, L. C., Pinto, J. C. \& Vieira, M. (2015). Construção da Carreira: O Papel da Perceção dos Filhos acerca dos Estilos Educativos Parentais na Exploração Vocacional.

0,01); Importância da Posição Preferida $(r=0,40 ; p<$ 0,01). E, ainda se verifica a presença de uma correlação positiva forte entre a subescala Instrumentalidade Interna e a Importância da Profissão Preferida $(r=0,23 ; p<0,01)$.

A dimensão da exigência apresenta uma correlação positiva e estatisticamente significativa com as subescalas:
Estatuto do Emprego, $(r=0,15 ; p<0,05)$, Certeza nos Resultados de Exploração $(r=0,17 ; p<0,01)$; Instrumentalidade Interna $(r=0,19 ; p<0,01)$.

A dimensão de responsividade não está associada de modo estatisticamente significativo com nenhuma das subescalas das crenças de exploração vocacional.

Tabela 2

Matriz das Correlações entre as Crenças da Exploração Vocacional e as Dimensões de Exigência e Responsividade $(n=296)$

\begin{tabular}{lccccccc}
\hline & EE & CRE & IE & II & IPP & Exigência & $\begin{array}{c}\text { Responsi- } \\
\text { vidade }\end{array}$ \\
\hline Estatuto de Emprego (EE) & 0,81 & $0,50^{* *}$ & $0,42^{* *}$ & $0,32^{* *}$ & $0,23^{* *}$ & $0,15^{*}$ & 0,06 \\
Certeza nos Resultados de Exploração (CRE) & & 0,87 & $0,33^{* *}$ & $0,19^{* *}$ & $0,29^{* *}$ & $0,17^{* *}$ & 0,06 \\
Instrumentalidade Externa (IE) & & & 0,82 & $0,66^{* *}$ & $0,40^{* *}$ & $0,19^{* *}$ & 0,07 \\
Instrumentalidade Interna (II) & & & 0,79 & $0,23^{* *}$ & 0,10 & $-0,02$ \\
Importância da Profissão Preferida (IPP) & & & & 0,80 & 0,11 & 0,11 \\
Exigência & & & & & & 0,54 & $0,24^{* *}$ \\
Responsividade & & & & & & & 0,86 \\
\hline
\end{tabular}

$* p<0,05 ; * p<0,01$.

A leitura da Tabela 3 permite identificar a presença de uma correlação positiva forte entre a subescala Exploração do Meio e as subescalas de Exploração de Si Próprio $(r=$ $0,41 ; p<0,01)$; Exploração Sistemática e Intencional $(r=$ $0,38 ; p<0,01)$; Quantidade da Informação $(r=0,39 ; p<$ $0,01)$. Verifica-se a presença de uma correlação positiva forte entre a subescala e a Exploração de Si Próprio e as subescalas Exploração Sistemática e Intencional $(r=0,19$; $p<0,01)$; Quantidade da Informação $(r=0,15 ; p<0,01)$. Verifica-se também, a presença de uma correlação positiva forte entre a subescala Exploração Sistemática e Intencional e a Quantidade da Informação $(r=0,32 ; p<0,01)$.

A dimensão da exigência apresenta uma correlação positiva estatisticamente significativa com as subescalas: Exploração do Meio $(r=0,21 ; p<0,01)$; Exploração de $\mathrm{Si}$ Próprio $(r=0,13 ; p<0,05)$; e Quantidade da Informação $(r=0,17 ; p<0,01)$.

As dimensões de responsividade não estão associadas de modo estatisticamente significativo com nenhuma das subescalas dos comportamentos de exploração vocacional.

Tabela 3

Correlações entre os Comportamentos da Exploração Vocacional e as Dimensões de Exigência e Responsividade

\begin{tabular}{lcccccc}
\hline & EM & ESP & ESI & QI & Exigência & Responsividade \\
\hline Exploração do Meio (EM) & 0,70 & $0,41^{* *}$ & $0,38^{* *}$ & $0,39^{* *}$ & $0,21^{* *}$ & 0,06 \\
Exploração de Si Próprio (ESP) & & 0,71 & $0,19^{* *}$ & $0,15^{* *}$ & $0,13^{*}$ & 0,05 \\
Exploração Sistémica Intencional (ESI) & & & 0,54 & $0,32^{* *}$ & 0,11 & 0,04 \\
Quantidade de Informação (QI) & & & & 0,69 & $0,17^{* *}$ & 0,02 \\
Exigência & & & & 0,54 & $0,24 * *$ \\
Responsividade & & & & & 0,86 \\
\hline
\end{tabular}

$* p<0,05 ; * * p<0,01$. 
A leitura da Tabela 4 permite identificar a presença de uma correlação positiva forte entre a subescala Stresse com a Exploração e a subescala de Stresse com a Decisão $(r=0,56 ; p<0,01)$.
A dimensão da exigência apresenta uma correlação positiva estatisticamente significativa com a subescala da Satisfação com a Informação $(r=0,15 ; p<0,01)$.

A dimensão de responsividade não está associada de modo estatisticamente significativo com nenhuma das subescalas das reações de exploração vocacional.

Tabela 4

Correlações entre as Reações da Exploração Vocacional e as Dimensões de Exigência e Responsividade

\begin{tabular}{lccccc}
\hline & SI & SE & SD & Exigência & Responsividade \\
\hline Satisfação com a Informação (SI) & 0,77 & 0,10 & $-0,74$ & $0,15^{*}$ & 0,08 \\
Stresse com a Exploração (SE) & & 0,81 & $0,56^{*}$ & 0,05 & $-0,02$ \\
Stresse com a Decisão (SD) & & & 0,88 & 0,03 & $-0,02$ \\
Exigência & & & 0,54 & $0,24^{*}$ \\
Responsividade & & & & 0,86
\end{tabular}

${ }^{*} p<0,01$.

\section{Discussão}

Neste ponto discutem-se e interpretam-se os resultados obtidos no estudo empírico, tendo como suporte a revisão da literatura subjacente a esta investigação, de forma a derivar implicações para o envolvimento parental na intervenção em orientação profissional com jovens.

No que diz respeito às subescalas da exploração vocacional, de uma forma geral, obtiveram-se melhores resultados para as participantes do sexo feminino, em particular no que diz respeito à Instrumentalidade Externa e Interna e à Exploração de Si Próprias. Isto é, as participantes do sexo feminino possuem crenças mais elevadas em como a exploração de si próprias e a exploração do mundo académico e profissional poderá favorecer a obtenção dos seus objetivos de carreira. Por este motivo, apresentam também mais comportamentos de exploração de si. Estes resultados são congruentes com outros estudos que referem que são as participantes do sexo feminino que mais exploram (Mota, 2010). Esta diferenciação da exploração vocacional associada ao sexo poderá ser explicada pela relação entre a atividade exploratória e a aprendizagem académica, que se revela mais intensa e bem-sucedida entre as jovens do sexo feminino (Noack et al., 2010). É possível, ainda, que a menor exploração vocacional por parte dos rapazes, reiterando estudos prévios (Faria, 2008; Noack et al., 2010), possa em parte ser explicada pelo maior impacto do estilo educativo responsividade junto daqueles adolescentes, ou seja, pelas atitudes de compreensão, de apoio emocional e de comunicação bidirecional dos pais para com os filhos (Tatsch, Teixeira, \& Gomes, 2003).

Esta constatação reflete o equilíbrio instável entre trabalho-família, apontado por Savickas (2012) como um aspeto merecedor de atenção para a compreensão das competências e das aspirações percecionadas e valorizadas.
Tal situação requer um aconselhamento dirigido para o life design da pessoa, isto é, para a maneabilidade dos diversos recursos pessoais e contextuais, e para a adaptabilidade às mudanças ao longo do percurso da vida.

As participantes do sexo feminino percecionam os estilos educativos dos seus pais com características mais exigentes, ou seja, como impondo e fazendo cumprir regras e normas sociais no contexto familiar e social (e.g., Weber, Prado, Viezzer, \& Brandenburg, 2004). Vignoli et al. (2005) apontam a vinculação aos pais como estando mais relacionada com a exploração de carreira das participantes do sexo feminino do que a dos participantes do sexo masculino, mas a explicação do processo subjacente está longe de estar clarificada.

A hipótese dos estilos educativos parentais percecionados se relacionarem com os níveis de exploração vocacional dos adolescentes confirmou-se relativamente ao estilo educativo parental exigência, que se correlacionou positiva e significativamente com o Estatuto do Emprego, Certeza nos Resultados de Exploração, Instrumentalidade Interna, Exploração do Meio, Exploração de Si Próprio, Quantidade da Informação e, Satisfação com a Informação. Ou seja, os alunos que percecionam os seus pais como mais exigentes são os que possuem crenças mais elevadas acerca da utilidade do comportamento exploratório e, efetivamente realizam mais comportamentos neste âmbito. Este dado é convergente com os de Blustein et al. (1991), Dietrich e Kracke (2009) e de Noack et al. (2010), que revelaram uma associação positiva entre pais que controlam, supervisionam e disciplinam o comportamento dos filhos, e a segurança na exploração de si e da realidade dos filhos, bem como com a disponibilidade para fazerem escolhas vocacionais mais consistentes e com percursos formativos mais longos e exigentes dos filhos. Estes resultados podem ser, igualmente, analisados à luz das proposições da teoria da 
Faria, L. C., Pinto, J. C. \& Vieira, M. (2015). Construção da Carreira: O Papel da Perceção dos Filhos acerca dos Estilos Educativos Parentais na Exploração Vocacional.

construção da carreira (Savickas, 2005), designadamente a dinâmica complexa entre a pessoa e o seu ecossistema, fazendo com que tanto as barreiras como as oportunidades/ recursos do contexto estejam na base da adaptação mútua ao longo do tempo. Esta situação pressupõe a ausência de condições controladas que possam ser antecipadas e, como tal, a imprevisibilidade dos percursos de vida. A carreira deve ser vista como um processo longitudinal de adaptação aos vários contextos e situações de vida, mais centrado no indivíduo e no seu conhecimento e identidade, enquanto produtos de interação social e de contínua co-construção de significação (Savickas, 2012).

Assim, poder-se-á antecipar que pais que garantem um contexto familiar em que os filhos sentem a supervisão e o estabelecimento de regras, e a imposição de alguns limites das figuras parentais, proporcionarão uma maior gama de oportunidades de exploração vocacional (Blustein, Prezioso, \& Schultheiss, 1995). Neste sentido, a carreira é assumida como o resultado de uma construção pessoal, fruto da interação continuada entre um conjunto de variáveis individuais, contextuais e relacionais, que podem ser trabalhadas e modificadas tendo em vista a promoção da capacidade de adaptação a um mundo em constante mudança (Pinto, 2010; Savickas, 2005).

\section{Conclusão}

Embora este estudo se tenha limitado a avaliar a influência dos estilos educativos parentais na exploração vocacional, a partir das perceções dos adolescentes, pelo recurso a uma metodologia quantitativa, e a amostra não seja representativa, o que não nos permite generalizações dos resultados, poderemos concluir que os estilos educativos parentais percecionados têm um papel na exploração de carreira e no desenvolvimento vocacional. Os dados exploratórios discutidos previamente justificam a prossecução dos estudos neste domínio, pois apontam dados consonantes com estudos prévios realizados no âmbito da teoria da construção da carreira relativamente a outros domínios do desenvolvimento pessoal (Savickas, 2011, 2012).

Em estudos futuros sugere-se a replicação deste estudo junto de um grupo mais vasto de participantes e em momentos diferentes da sua exploração vocacional, como por exemplo no início de um curso (regular ou profissional) e depois no final, quando o aluno tiver concluído o $12^{\circ}$ ano do mesmo curso. Para o conceito de exploração vocacional é também pertinente para possíveis investigações futuras, o estudo das influências dos professores e do grupo de pares, uma vez que ambos são considerados agentes fundamentais para a construção do self do jovem, e desta forma, devem ser contemplados no processo de exploração vocacional em estudos futuros. Sugere-se ainda a realização de programas com pais para assumirem maior protagonismo neste processo de desenvolvimento vocacional (Kush \& Cochran, 1993), na tentativa de perceber como os pais podem ser agentes ativos do desenvolvimento vocacional, promovendo a autonomia dos filhos.

Estes resultados sugerem a importância dos profissionais de psicologia, poderem desenvolver intervenções de orientação profissional, que visem o envolvimento parental na exploração vocacional dos seus filhos (e.g., Faria, 2008; Gonçalves, 2008). A confirmação neste estudo, da influência percecionada pelos jovens dos estilos educativos parentais no processo de exploração vocacional, coloca o repto aos profissionais de psicologia, que desenvolvem a sua ação em estudos e projetos no âmbito da orientação profissional, para reformularem a sua intervenção em orientação profissional, uma vez que o contexto familiar é relevante para o desenvolvimento vocacional do jovem (e.g. Gonçalves, 2008). Os pais poderão envolver-se em interações intencionais com os seus filhos adolescentes, no sentido de promover experiências de exploração vocacional. Por exemplo, os pais podem encorajar, um adolescente a participar num clube desportivo para, intencionalmente, promover uma atitude de competição frente à vida, ou então levar uma filha adolescente a visitar um campus universitário para que esta possa considerar a possibilidade de investir num curso superior (Young, Valach, Ball, Turkel, \& Wong, 2003), ou ainda, explorar conjuntamente informação, em papel ou em suporte informático, sobre os sistemas de formação e profissional, ajudando os filhos a lidarem com os seus múltiplos questionamentos acerca do seu projeto vocacional em configuração.

\section{Referências}

Baumrind, D. (1971). Current patterns of parental authority. Developmental Psychology Monographs, 4, 1-103. doi:10.1037/ h0030372

Baumrind, D. (1989). Rearing competent children. In W. Danon (Ed.), Child development today and tomorrow (pp. 349-378). San Francisco, CA: Jossey Bass.

Berríos-Allison, A. C. (2005). Family influences on college students' occupational identity. Journal of Career Assessment, 13(2), 233-247. doi:10.1177/1069072704270320

Blustein, D. L., Prezioso, M. S., \& Schultheiss, D. P. (1995). Attachment theory and career development: Current status and future directions. The Counseling Psychologist, 23, 416-432. doi:10.1177/0011000095233002

Blustein, D. L., Walbridge, M. M., Friedlander, M. L., \& Palladino, D. E. (1991). Contributions of psychological separation and parental attachment to the career development process. Journal of Counseling Psychology, 38(1), 39-50.

Bryant, B. K., Zvonkovic, A. M., \& Reynolds, P. (2006). Parenting in relation to child and adolescent vocational development. Journal of Vocational Behaviour, 69, 149-175. doi:10.1016/j. jvb.2006.02.004

Carvalho, M. S. (2007). Perspectivas sobre a influência parental na execução de planos de carreira no Ensino Secundário (Dissertação de mestrado, Escola de Psicologia, Universidade do Minho, Braga, Portugal). Recuperado em http://repositorium. sdum.uminho.pt/handle/1822/9766

Costa, F. T., Teixeira, M. A. P., \& Gomes, W. B. (2000). Responsividade e exigência: Duas escalas para avaliar estilos 
parentais. Psicologia: Reflexão e Crítica,13(3), 465-473. doi:10.1590/S0102-79722000000300014

Darling, N., \& Steinberg, L. (1993). Parenting style as context: An integrative model. Psychological Bulletin, 113, 487-496. doi:10.1037/0033-2909.113.3.487

Dietrich, J., \& Kracke, B. (2009). Career-specific parental behaviors in adolescents' development. Journal of Vocational Behavior, 75, 109-119. doi:10.1016/j.jvb.2009.03.005

Ducharne, M., Cruz, O., Marinho, S., \& Grande, C. (2006). Questionário de Estilos Educativos Parentais. Psicologia e Educação, 1, 63-75.

Faria, L. (2008). A eficácia da consulta psicológica vocacional de jovens - Estudo do impacto de uma intervenção (Tese de doutorado, Instituto de Educação e Psicologia, Universidade do Minho, Braga, Portugal). Recuperado em http://repositorium.sdum.uminho.pt/handle/1822/8502

Fouad, N. A., Kantamneni, N., Smothers, M., K., Chen, Y. L., Fitzpatrick, M. E., Guillen, A., \& Terry, S. (2008). Asian American Career Development: A qualitative analysis. Journal of Vocational Behavior, 72, 43-59. doi:10.1016/j. jvb.2007.10.002

Galinsky, E. (2000). Ask the children: The breakthrough study that reveals how to succed at work and parenting. New York: Quill.

Gonçalves, C. M. (2006). A família e a construção de projetos vocacionais de adolescentes e jovens (Tese de doutorado não-publicada, Faculdade de Psicologia e de Ciências de Educação, Universidade do Porto, Portugal).

Gonçalves, C. M. (2008). Pais aflitos, filhos com futuro incerto? - Um estudo sobre a influência das famílias na orientação dos filhos. Lisboa, Portugal: Fundação Calouste Gulbenkian.

Holland, J. L. (1992). Making vocational choices: A theory of vocational personalities and work environments ( $\left.2^{\text {nd }} \mathrm{ed}.\right)$. Odessa, FL: Psychological Assessment Resources.

Hutz, C. S., \& Bardagi, M. P. (2006). Indecisão profissional, ansiedade e depressão na adolescência: A influência dos estilos parentais. Psico-USF, 11(1), 65-73.

Kohn, M. (1977). Class and conformity. Chicago, IL: University of Chicago Press.

Kracke, B. (1997). Parental behaviors and adolescents' Career exploration. The Career Development Quarterly, 45, 341-350. doi:10.1002/j.2161-0045.1997.tb00538.x

Kush, K., \& Cochran, L. (1993). Enhancing a sense of agency through career planning. Journal of Counseling Psychology, 40, 434-439. doi:10.1037/0022-0167.40.4.434

Lamborn, S. D., Mounts, N. S., Steinberg, L., \& Dornbusch, S. M. (1991). Patterns of competence and adjustment among adolescents from authoritative, authoritarian, indulgent, and neglectful families. Child Development, 62, 1049-1065. doi:10.1111/j.1532-7795.2006.00119.x.

Maccoby, E., \& Martin, J. (1983). Socialization in the context of the family: Parent-child interaction. In P. H. Mussen \& E. Hetherington (Eds.), Handbook of child psychology: Socialization, personality and social development (pp. 1-101). New York: Wiley.

McAdams, D. P., \& Olson, B. D. (2010). Personality development: Continuity and change over the life course. Annual Review of Psychology, 61, 517-542. doi:10.1146/annurev. psych.093008.100507

Mortimer, J. T. (1976). Social class, work of family: Some implications of the father's occupation for family relationships and son's career decisions. Journal of Marriage and the Family, 38, 241-256.
Mota, A. I. B. (2010). Exploração, dificuldades de tomada de decisão e indecisão vocacional no Ensino Básico (Dissertação de mestrado, Escola de Psicologia, Universidade do Minho, Braga, Portugal). Recuperado em http://repositorium.sdum. uminho.pt/handle/1822/13456

Noack, P., Kracke, B., Gniewosz, B., \& Dietrich, J. (2010). Parental and school effects on student's occupational exploration: A longitudinal and multilevel analysis. Journal of Vocational Behavior, 77, 50-57. doi:10.1016/j.jvb.2010.02.006

O'Brien, K. M. (1996). The influence of psychological separation and parental attachment on the career development of adolescent women. Journal of Vocational Behavior, 48, 257-274. doi:10.1006/jvbe.1996.0024

Otto, A. R., Markman, A. B., Gureckis, T. M., \& Love, B. C. (2010). Regulatory fit and systematic exploration in a dynamic decision-making environment. Journal of Experimental Psychology: Learning, Memory, and Cognition, 36(3), 797-804. doi:10.1037/a0018999

Otto, L. B. (2000). Youth perspectives on parental career influence. Journal of Career Development, 27(2), 111-118. doi:10.1177/089484530002700205

Penick, N., \& Jepsen, D. (1992). Family functioning and adolescent career development. The Career Development Quarterly, 35, 71-76. doi:10.1002/j.2161-0045.1992.tb00327

Pinto, H. R., \& Soares, M. C. (2002). Influência parental no desenvolvimento vocacional dos adolescentes. Revista Portuguesa de Psicologia, 36, 111-137.

Pinto, J. C. (2010). Gestão pessoal da carreira: Estudo de um modelo de intervenção psicológica com bolseiros de investigação (Tese de doutorado, Universidade do Minho, Braga, Portugal). Recuperado em http://repositorium.sdum.uminho. pt/handle/1822/11633

Savickas, M. L. (2002). Career construction: A developmental theory of vocational behavior. In D. Brown (Ed.), Career choice and development (pp. 149-205). San Francisco, CA: Jossey-Bass.

Savickas, M. L. (2005). The theory and practice of career construction. In D. Brown \& R. Lent (Eds.), Career development and counselling: Putting theory and research to work (pp. 43-70). New York: John Wiley \& Sons.

Savickas, M. L. (2011). The self in vocational psychology: Object, subject, and project. In P. J. Hartung \& L. M. Subich (Eds.), Developing self in work and career: Concepts, cases, and contexts (pp. 17-33). Washington, DC: American Psychological Association Press.

Savickas, M. L. (2012). Life design: A paradigm for career intervention in the 21 st century. Journal of Counseling and Development, 90, 13-19. doi:10.1111/j.1556-6676.2012.00002.x

Schulenberg, J. E., Vondracek, F. W., \& Crouter, A. (1984). The influence of the family on vocational development. Journal of Marriage and the Family, 46, 129-143.

Schultheiss, D., \& Blustein, D. (1994). Contributions of family relationship factors to the identity formation process. Journal of Counseling and Development, 73(2), 159-166. doi:10.1002/j.1556-6676.1994.tb01729.x

Scott, D. J., \& Church, A. T. (2001). Separation/attachment theory and career decidedness and commitment: Effects of parental divorce. Journal of Vocational Behavior, 58, 328347. doi:10.1006/jvbe.2000.1769

Stumpf, S. A., Colarelli, S. M., \& Hartman, K. (1983). Development of the Career Exploration Survey (CES). Journal of Vocational Behavior, 22, 191-226. doi:10.1016/00018791(83)90028-3 
Faria, L. C., Pinto, J. C. \& Vieira, M. (2015). Construção da Carreira: O Papel da Perceção dos Filhos acerca dos Estilos Educativos Parentais na Exploração Vocacional.

Tatsch, D. T., Teixeira, M. A. P., \& Gomes, W. B. (2003). Estilos parentais na percepção dos adolescentes de comunidades ítalo e teuto-gaúchas. Mudanças, 34, 389-406.

Taveira, M. C. (1997). Exploração e desenvolvimento vocacional de jovens. Estudo sobre as relações entre a exploração, a identidade e a indecisão vocacional (Tese de doutorado, Instituto de Educação e Psicologia, Universidade do Minho, Braga, Portugal). Recuperado em http://repositorium.sdum. uminho.pt/handle/1822/19856

Teixeira, M. A. P., Bargadi, M. P., \& Gomes, W. B. (2004). Refinamento de um instrumento para avaliar responsividade e exigência parental percebidas na adolescência. Avaliação Psicológica, 3(1), 1-12.

Vignoli, E., Croity-Belz, S., Chapeland, V., Fillipis, A., \& Garcia, M. (2005). Career exploration in adolescents: The role of anxiety, attachment, and parenting style. Journal of Vocational Behavior, 67, 153-168. doi:10.1016/j.jvb.2004.08.006

Way, W. L., \& Rossmann, M. M. (1996). Learning to work: How parents nurture the transition from school to work. Family matter in school to work transition. Berkeley, CA: National Center for Research in Vocational Education.

Weber, L. N. D., Prado, P. M., Viezzer, A. P., \& Brandenburg, O. J. (2004). Identificação de estilos parentais: O ponto de vista dos pais e dos filhos. Psicologia: Reflexão e Crítica, 17(3), 323-331.

Whiston, S. C., \& Keller, B. K. (2004). The influence of the family of origin on career development: A review and analysis. Journal of Vocational Behavior, 32, 493-568. doi:10.1177/0011000004265660

Young, R. A., Marshall, S., Domene, J. F., Arato-Bolivar, J., Hayoun, R., Marshall, E., ... Valach, L. (2005). Relationships, communication, and career in the parent-adolescent projects of families with and without challenges. Journal of Vocational Behaviour, 68(1), 1-23. doi:10.1016/j.jvb.2005.05.001

Young, R. A., Valach, L., Ball, J., Turkel, H., \& Wong, Y. S. (2003). The family career development project in Chinese Canadian families. Journal of Vocational Behavior, 62, $287-$ 304. doi:10.1016/S0001-8791(02)00022-2 